\title{
ON A TWO-DIMENSIONAL ELLIPTIC PROBLEM WITH LARGE EXPONENT IN NONLINEARITY
}

\author{
XIAOFENG REN AND JUNCHENG WEI
}

\begin{abstract}
A semilinear elliptic equation on a bounded domain in $R^{2}$ with large exponent in the nonlinear term is studied in this paper. We investigate positive solutions obtained by the variational method. It turns out that the constrained minimizing problem possesses nice asymptotic behavior as the nonlinear exponent, serving as a parameter, gets large. We shall prove that $c_{p}$, the minimum of energy functional with the nonlinear exponent equal to $p$, is like $(8 \pi e)^{1 / 2} p^{-1 / 2}$ as $p$ tends to infinity.

Using this result, we shall prove that the variational solutions remain bounded uniformly in $p$. As $p$ tends to infinity, the solutions develop one or two peaks. Precisely the solutions approach zero except at one or two points where they stay away from zero and bounded from above.

Then we consider the problem on a special class of domains. It turns out that the solutions then develop only one peak. For these domains, the solutions enlarged by a suitable quantity behave like a Green's function of $-\Delta$. In this case we shall also prove that the peaks must appear at a critical point of the Robin function of the domain.
\end{abstract}

\section{INTRODUCTION}

Studies concerning asymptotic behavior of elliptic nonlinear partial differential equations have grown rapidly in recent years. Among many delicate results, we would like to mention one obtained independently by Han [7] and Rey [12] through different approaches. They considered the following semilinear elliptic problem on a smooth bounded domain $\Omega$ in $R^{n}$ with $n \geq 3$ :

$$
\left\{\begin{array}{l}
\Delta u+u^{p}=0 \quad \text { in } \Omega \subset R^{n}, \quad n \geq 3, \\
\left.u\right|_{\partial \Omega}=0 .
\end{array}\right.
$$

They showed that when $p$ approaches $(n+2) /(n-2)$, the well-known critical exponent, the positive solutions obtained by the variational method will blow up at some point $x_{0}$ which is a critical point of function $R$ where $R(x)=g(x, x)$, and $g(x, y)$ is the regular part of the Green's function of $-\Delta$.

Received by the editors November 20, 1992.

1991 Mathematics Subject Classification. Primary 35B40, 35A08, 35A15; Secondary 34A34, $46 \mathrm{E} 35$. 
In this paper we consider the same equation on a smooth bounded domain in $R^{2}$

$$
\left\{\begin{array}{l}
\Delta u+u^{p}=0 \quad \text { in } \Omega \subset R^{2}, \\
\left.u\right|_{\partial \Omega}=0
\end{array}\right.
$$

This time because the exponent can be arbitrarily large for (1.2) to have a positive variational solution, we impose the following questions.

Question 1. If we take $p$ to be a parameter, do solutions obtained by variational method, specified later, blow up when $p$ approaches infinity?

Question 2. What do the normalized solutions, specified later, look like when $p$ approaches infinity?

It turns out that the answer to the first question surprisingly differs from the one in higher dimensional case while the answer to the second question remains similar to the one in higher dimensional case for some domains. To state our results precisely we need a few words on the solutions to (1.2). Since a complete classification of the solutions to (1.2) is still open, we shall focus on the solutions to (1.2) obtained by the following variational method. These solutions exist for any $p$ provided $\Omega$ is bounded and smooth enough.

Consider the constrained minimizing problem

$$
\inf \left\{\int_{\Omega}|\nabla u|^{2}: u \in W_{0}^{1,2}(\Omega),\|u\|_{p+1}=1\right\}:=c_{p}^{2} .
$$

A standard variational argument shows that $c_{p}^{2}$ can be achieved by a positive function, say $u_{p}^{\prime}$, in the desired class. Then a positive scalar multiple of $u_{p}^{\prime}$, say $u_{p}$, solves $(1.2)$ and

$$
c_{p}=\left\|\nabla u_{p}\right\|_{L^{2}} /\left\|u_{p}\right\|_{L^{p+1}} .
$$

From now on throughout the rest of this article we denote solutions obtained in this way by $u_{p}$. Our first result is

Theorem 1.1. There exist $C_{1}, C_{2}$, independent of $p$, such that $0<C_{1}<$ $\left\|u_{p}\right\|_{L^{\infty}}<C_{2}<\infty$ for $p$ large enough.

Theorem 1.1 shows a substantial difference between (1.1) and (1.2). It also shows that the peak set of $u_{p}$ is nonempty. By a point $P$ being in the peak set of $u_{p}$, we mean that $u_{p}$ does not vanish in $L^{\infty}$ norm in any small neighborhood of $P$ as $p \rightarrow \infty$. To state further results, we need some definitions.

Let

$$
v_{p}=u_{p} / \int_{\Omega} u_{p}^{p}
$$

For a sequence $v_{p_{n}}$ of $v_{p}$ we define the blow-up set $S$ of $v_{p_{n}}$ to be the subset of $\bar{\Omega}$ such that $x \in S$ if there exist a subsequence, still denoted by $v_{p_{n}}$, and a sequence $x_{n}$ in $\Omega$ with

$$
v_{p_{n}}\left(x_{n}\right) \rightarrow \infty \text { and } x_{n} \rightarrow x
$$


We shall use $\# S$ to denote the cardinality of $S$. It will be shown later that the blow-up set $S$ of $v_{p_{n}}$ contains the peak set of $u_{p_{n}}$.

We say that a smooth bounded domain $\Omega$ has condition $(\mathrm{T})$ if there exists a point $y$ in $\Omega$ such that

(1) $\Omega$ is star-shaped with respect to $y$, i.e., $(x-y, n(x))>0$ for all $x \in$ $\partial \Omega$

$$
\int_{\partial \Omega} \frac{d s}{(x-y, n(x))}<2 \pi e
$$

where $n(x)$ denotes the outer normal of $\partial \Omega$ at $x$.

Theorem 1.2. For any smooth bounded domain $\Omega$ and a sequence $v_{p_{n}}$ of $v_{p}$ with $p_{n} \rightarrow \infty$ there exists a subsequence of $v_{p_{n}}$ such that the blow-up set $S$ of that subsequence is contained in $\Omega$ and has the property $1 \leq \# S \leq 2$; in other words the subsequence must blow up and it blows up at most at two points in $\Omega$.

If $\Omega$ meets condition $(\mathrm{T})$, then the above subsequence must blow up at one point in $\Omega$.

In the second case of the above theorem, we have a more delicate description; especially we can locate the blow-up point, i.e., peak point.

Theorem 1.3. Let $\Omega$ be a smooth bounded domain with condition $(\mathrm{T})$. Then for any sequence $v_{p_{n}}$ of $v_{p}$ with $p_{n} \rightarrow \infty$ there exists a subsequence of $v_{p_{n}}$, still denoted by $v_{p_{n}}$, such that

(1)

$$
f_{n}:=\frac{u_{p_{n}}^{p_{n}}}{\int_{\Omega} u_{p_{n}}^{p_{n}}}=\left(\int_{\Omega} u_{p_{n}}^{p_{n}}\right)^{p_{n}-1} v_{p_{n}}^{p_{n}} \rightarrow \delta\left(x_{0}\right)
$$

in the sense of distribution where $\delta\left(x_{0}\right)$ is the $\delta$ function at point $x_{0}$ and $\left\{x_{0}\right\}=S$.

(2) $v_{p_{n}} \rightarrow G\left(x, x_{0}\right)$ in $W^{1, q}(\Omega)$ weakly for any $1<q<2$ where $G$ is the Green's function of $-\Delta$ on $\Omega$; furthermore for any compact subset $K$ of $\bar{\Omega} \backslash\left\{x_{0}\right\}$ we have $v_{p_{n}} \rightarrow G\left(x, x_{0}\right)$ in $C^{2, \alpha}(K)$.

(3) $x_{0}$ is a critical point of the Robin function $R$ where $R(x)=g(x, x)$ and

$$
g(x, y)=G(x, y)+\frac{1}{2 \pi} \log |x-y|
$$

is the regular part of the Green's function $G$.

From the above results, we can see that when $p$ gets large, the solutions $u_{p}$ obtained by the variational method look more and more like a single or double spike. This kind of asymptotic behavior also arises from other problems. Here we only mention one result obtained by $\mathrm{Ni}$ and Takagi in the study of a biological pattern formation problem. We refer to [9] for details and further references.

Our paper is organized as follows. In $\S 2$, we prove a crucial estimate on $c_{p}$ defined in (1.3). Then we prove Theorem 1.1 in $\S 3$, Theorem 1.2 in $\S 4$, and Theorem 1.3 in $\S 5$. We also include some thoughts in the last section. 


\section{Some estimates}

Recall $c_{p}$ defined in (1.3). We state a lemma from our earlier work [11] with a brief proof.

Lemma 2.1. For every $t \geq 2$ there is $D_{t}$ such that $\|u\|_{L^{t}} \leq D_{t} t^{1 / 2}\|\nabla u\|_{L^{2}}$ for all $u \in W_{0}^{1,2}(\Omega)$ where $\Omega$ is a bounded domain in $R^{2}$; furthermore

$$
\lim _{t \rightarrow \infty} D_{t}=(8 \pi e)^{-1 / 2} \text {. }
$$

Proof. Let $u \in W_{0}^{1,2}(\Omega)$. We know $x^{s} / \Gamma(s+1) \leq e^{x}$ for all $x \geq 0, s \geq 0$ where $\Gamma$ is the $\Gamma$ function. From a sharp form of the Trudinger's Inequality (see [6, p. 160] and [8]), we have

$$
\int_{\Omega} \exp \left[4 \pi\left(\frac{u}{\|\nabla u\|_{L^{2}}}\right)^{2}\right] d x \leq C|\Omega|
$$

where $C$ does not depend on anything and $|\Omega|$ is the Lebesgue measure of $\Omega$. Therefore

$$
\begin{aligned}
\frac{1}{\Gamma(t / 2+1)} \int_{\Omega} u^{t} d x & =\frac{1}{\Gamma(t / 2+1)} \int_{\Omega}\left[4 \pi\left(\frac{u}{\|\nabla u\|_{L^{2}}}\right)^{2}\right]^{t / 2} d x(4 \pi)^{-t / 2}\|\nabla u\|_{L^{2}}^{t} \\
& \leq \int_{\Omega} \exp \left[4 \pi\left(\frac{u}{\|\nabla u\|_{L^{2}}}\right)^{2}\right] d x(4 \pi)^{-t / 2}\|\nabla u\|_{L^{2}}^{t} \\
& \leq C|\Omega|(4 \pi)^{-t / 2}\|\nabla u\|_{L^{2}}^{t} .
\end{aligned}
$$

Hence

$$
\left(\int_{\Omega} u^{t} d x\right)^{1 / t} \leq\left(\Gamma\left(\frac{t}{2}+1\right)\right)^{1 / t} C^{1 / t}(4 \pi)^{-1 / 2}|\Omega|^{1 / t}\|\nabla u\|_{L^{2}(\Omega)} .
$$

Notice that, according to Stirling's formula,

$$
\left(\Gamma\left(\frac{t}{2}+1\right)\right)^{1 / t} \sim\left(\left(\frac{t / 2}{e}\right)^{t / 2} \sqrt{t e} e^{\theta_{t}}\right)^{1 / t} \sim\left(\frac{1}{2 e}\right)^{1 / 2} t^{1 / 2}
$$

where $0<\theta_{t}<\frac{1}{12}$. Choosing $D_{t}$ to be

$$
(\Gamma(t / 2+1))^{1 / t} C^{1 / t}(4 \pi)^{-1 / 2}|\Omega|^{1 / t} t^{-1 / 2}
$$

we get the desired result.

In [11] we have an estimate for a quantity similar to $c_{p}$. Here we need a finer result.

Lemma 2.2.

$$
\lim _{p \rightarrow \infty} \frac{c_{p}}{p^{-1 / 2}}=(8 \pi e)^{1 / 2}
$$

Proof. Without loss of generality, we assume $0 \in \Omega$. Let $L>0$ be such that $B_{L} \subset \Omega$ where $B_{L}$ is the ball of radius $L$ centered at origin. For $0<l<L$ 
consider Moser's function

$$
m_{l}(x)=\frac{1}{\sqrt{2 \pi}} \begin{cases}(\log (L / l))^{1 / 2}, & 0 \leq|x| \leq l \\ \frac{\log (L /|x|)}{[\log (L / l)]^{1 / 2},} & l \leq|x| \leq L \\ 0, & |x| \geq L .\end{cases}
$$

Then $m_{l} \in W_{0}^{1,2}(\Omega)$ and $\|\nabla u\|_{L^{2}}=1$. Now

$$
\begin{aligned}
\int_{\Omega} m_{l}^{p+1}(x) d x= & {\left[\frac{1}{\sqrt{2 \pi}}\left(\log \frac{L}{l}\right)^{1 / 2}\right]^{p+1}\left|B_{l}\right| } \\
& +\left[\frac{1}{\sqrt{2 \pi}}\left(\log \frac{L}{l}\right)^{-1 / 2}\right]^{p+1} \int_{l<|x|<L}\left(\log \frac{L}{|x|}\right)^{p+1} d x \\
:= & I_{1}+I_{2}, \\
I_{1}= & {\left[\frac{1}{\sqrt{2 \pi}}\left(\log \frac{L}{l}\right)^{1 / 2}\right]^{p+1} \pi l^{2}, } \\
I_{2}= & {\left[\frac{1}{\sqrt{2 \pi}}\left(\log \frac{L}{l}\right)^{-1 / 2}\right]^{p+1} \int_{l<|x|<L}\left(\log \frac{L}{|x|}\right)^{p+1} d x . }
\end{aligned}
$$

Choosing $l=L e^{-(p+1) / 4}$, we have

$$
\left\|m_{l}\right\|_{L^{p+1}} \geq I_{1}^{1 /(p+1)} \geq[1 / 8 \pi e]^{1 / 2}(p+1)^{1 / 2}\left(\pi L^{2}\right)^{1 /(p+1)} .
$$

Hence

$$
c_{p} \leq[8 \pi e]^{1 / 2}(p+1)^{-1 / 2}\left(\pi L^{2}\right)^{-1 /(p+1)} .
$$

Combining this with Lemma 2.1, we have

$$
\lim _{p \rightarrow \infty} \frac{c_{p}}{p^{-1 / 2}}=(8 \pi e)^{1 / 2}
$$

By the construction in $\S 1$ of our solutions $u_{p}$, we know

$$
c_{p}=\left\|\nabla u_{p}\right\|_{L^{2}} /\left\|u_{p}\right\|_{L^{p+1}} .
$$

Because we also have an integral identity

$$
\int_{\Omega}\left|\nabla u_{p}\right|^{2}=\int_{\Omega} u_{p}^{p+1}
$$

which can be easily derived by multiplying (1.2) by $u_{p}$ and integrating the equality, we have the following estimates.

Corollary 2.3.

$$
p \int_{\Omega} u_{p}^{p+1} \rightarrow 8 \pi e, \quad p \int_{\Omega}\left|\nabla u_{p}\right|^{2} \rightarrow 8 \pi e,
$$

as $p \rightarrow \infty$. 
We now define an important quantity:

$$
L_{0}=\varlimsup_{p \rightarrow \infty} \frac{p \nu_{p}}{e}
$$

where

$$
\nu_{p}=\int_{\Omega} u_{p}^{p}
$$

We have the following estimate for $L_{0}$.

Lemma 2.4. (1) For any smooth bounded domain $\Omega$ of $R^{2}, L_{0} \leq 8 \pi$.

(2) For smooth bounded domain with condition $(\mathrm{T}), L_{0}<8 \pi$.

Proof. Part (1) follows immediately from Hölder's Inequality and Corollary 2.3. We observe

$$
\begin{aligned}
\varlimsup_{p \rightarrow \infty} p \int_{\Omega} u_{p}^{p} & \leq \varlimsup_{p \rightarrow \infty} p\left(\int_{\Omega} u_{p}^{p+1}\right)^{p /(p+1)}|\Omega|^{1 /(p+1)} \\
& \leq \varlimsup_{p \rightarrow \infty} p\left(\frac{8 \pi e}{p}\right)^{p /(p+1)}|\Omega|^{1 /(p+1)} \leq 8 \pi e
\end{aligned}
$$

To prove part (2) we first state the well-known Pohozaev Identity [10]. Let $u$ solve $\Delta u+f(x, u)=0$ in $\Omega \in R^{N}$. Then we have

$$
\begin{aligned}
\int_{\Omega}[ & \left.N F(x, u)-\frac{N-2}{2} u f(x, u)+(x-y) F_{x}(x, u)\right] d x \\
=\int_{\partial \Omega}\left[(x-y, \nabla u) \frac{\partial u}{\partial n}-(x-y, n) \frac{|\nabla u|}{2}\right. & \left.+(x-y, n) F(x, u)+\frac{N-2}{2} u \frac{\partial u}{\partial n}\right] d s
\end{aligned}
$$

for all $y \in R^{N}$ where

$$
F(x, u)=\int_{0}^{u} f(x, t) d t
$$

Apply it to

$$
\Delta u+u^{p}=0 \quad \text { in } \Omega,\left.\quad u\right|_{\partial \Omega}=0 .
$$

Therefore assuming that $y$ is the point in the definition of condition (T) (without loss of generality we take $y$ to be 0 in $R^{2}$ ),

$$
\frac{2}{p+1} \int_{\Omega} u^{p+1}=\frac{1}{2} \int_{\partial \Omega}(x, n)\left(\frac{\partial u(x)}{\partial n(x)}\right)^{2} d s
$$

where $n(x)$ is the outer normal of $\partial \Omega$ at point $x$. If we integrate the equation directly, we also have

$$
\int_{\Omega} u^{p}=\int_{\partial \Omega} \frac{\partial u}{\partial n} d s
$$


Hence letting $u=u_{p}$, we have

$$
\begin{aligned}
\int_{\Omega} u_{p}^{p} & \leq\left(\int_{\partial \Omega} \frac{d s}{(x, n)}\right)^{1 / 2}\left(\int_{\partial \Omega}(x, n)\left(\frac{\partial u_{p}}{\partial n}\right)^{2} d s\right)^{1 / 2} \\
& \leq\left(\int_{\partial \Omega} \frac{d s}{(x, n)}\right)^{1 / 2}\left(\frac{4}{p+1} \int_{\Omega} u^{p+1}\right)^{1 / 2} .
\end{aligned}
$$

Therefore by condition $(\mathrm{T})$

$$
\begin{aligned}
\varlimsup_{p \rightarrow \infty} \frac{p \int_{\Omega} u^{p}}{e} & \leq\left(\int_{\partial \Omega} \frac{d s}{(x, n)}\right)^{1 / 2}(32 \pi e)^{1 / 2} e^{-1} \\
& <(2 \pi e)^{1 / 2}(32 \pi e)^{1 / 2} e^{-1}=8 \pi .
\end{aligned}
$$

This completes the proof of the lemma.

Remark 2.5. If $\Omega$ is a ball, then $\Omega$ satisfies condition ( $\mathrm{T})$ with $\int_{\partial \Omega} d s /(x, n)=$ $2 \pi$. So the class of $\Omega$ with condition $(\mathrm{T})$ is not very small.

\section{Proof of Theorem 1.1}

Now we get down to the proof of Theorem 1.1.

A uniform lower bound actually exists for any positive solutions to (1.2). Let $\lambda$ be the first eigenvalue of $-\Delta$ and $\varphi$ be a corresponding positive eigenfunction. Then if $u$ is any solution to (1.2) with the exponent equal to $p$, we have

$$
0=\int_{\Omega} u \Delta \varphi-\varphi \Delta u=-\lambda \int_{\Omega} u \varphi+\int_{\Omega} u^{p} \varphi .
$$

That is

$$
\int_{\Omega}\left(u^{p}-\lambda u\right) \varphi=0
$$

Hence

$$
\|u\|_{L^{\infty}(\Omega)} \geq \lambda^{1 /(p-1)} \rightarrow 1
$$

as $p \rightarrow \infty$ which yields a lower bound for large $p$.

To get a uniform upper bound for $u_{p}$, we let

$$
\gamma_{p}=\max _{x \in \bar{\Omega}} u_{p}(x), \quad \mathscr{A}=\left\{x: \gamma_{p} / 2<u_{p}(x)\right\}, \quad \Omega_{t}=\left\{x: t<u_{p}(x)\right\} .
$$

From Lemma 2.1 and Corollary 2.3

$$
\left(\int_{\Omega} u_{p}^{2 p}\right)^{1 /(2 p)} \leq C(2 p)^{1 / 2}\left\|\nabla u_{p}\right\|_{L^{2}} \leq M
$$

where $M$ is independent of $p$ if $p$ is large. Hence

$$
\left(\frac{\gamma_{p}}{2}\right)^{2 p}|\mathscr{A}| \leq \int_{\Omega} u_{p}^{2 p} \leq M^{2 p}
$$

On the other hand

$$
\int_{\Omega_{t}} u_{p}^{p}=-\int_{\Omega_{t}} \Delta u_{p}=\int_{\partial \Omega_{t}}\left|\nabla u_{p}\right| d s
$$




$$
-\frac{d}{d t}\left|\Omega_{t}\right|=\int_{\partial \Omega_{t}} \frac{d s}{\left|\nabla u_{p}\right|}
$$

where the second identity is called coarea formula. We refer to [4] for more information about this formula. Now by the Schwarz inequality and the isoperimetric inequality we have

$$
-\frac{d}{d t}\left|\Omega_{t}\right| \int_{\Omega_{t}} u_{p}^{p}(x) d x=\int_{\partial \Omega_{t}} \frac{d s}{\left|\nabla u_{p}\right|} \int_{\partial \Omega_{t}}\left|\nabla u_{p}\right| d s \geq\left|\partial \Omega_{t}\right|^{2} \geq 4 \pi\left|\Omega_{t}\right| .
$$

Define a function $r(t)$ such that $\left|\Omega_{t}\right|=\pi r^{2}(t)$; then

$$
\frac{d}{d t}\left|\Omega_{t}\right|=2 \pi r \frac{d r}{d t}
$$

Hence we have

$$
\begin{gathered}
-2 \pi r \frac{d r}{d t} \int_{\Omega_{t}} u_{p}^{p} \geq 4 \pi^{2} r^{2}, \quad-\frac{d r}{d t} \int_{\Omega_{t}} u_{p}^{p} \geq 2 \pi r, \\
-\frac{d t}{d r} \leq \frac{1}{2 \pi r} \int_{\Omega_{t}} u_{p}^{p} \leq \frac{1}{2 \pi r} \gamma_{p}^{p}\left|\Omega_{t}\right|=\frac{1}{2} r \gamma_{p}^{p} .
\end{gathered}
$$

Integrating the last inequality from 0 to $r_{0}$, we have

$$
t(0)-t\left(r_{0}\right) \leq \frac{1}{4} r_{0}^{2} \gamma^{p}
$$

Choosing $r_{0}$ so that $t\left(r_{0}\right)=\gamma_{p} / 2$ we get

$$
\begin{gathered}
\gamma_{p} \leq \frac{1}{2} r_{0}^{2} \gamma_{p}^{p} \\
\gamma_{p} \leq \frac{1}{2} r_{0}^{2} \gamma_{p}^{p}=\frac{1}{2 \pi}\left|\Omega_{\gamma_{p} / 2}\right| \gamma_{p}^{p}=\frac{1}{2 \pi}|\mathscr{A}| \gamma_{p}^{p} .
\end{gathered}
$$

From (3.1) and (3.2)

$$
\gamma_{p}^{2 p}|\mathscr{A}| \leq(2 M)^{2 p}, \quad \gamma_{p} \leq(1 / 2 \pi) \gamma_{p}^{p}\left(2 M / \gamma_{p}\right)^{2 p}
$$

which in turn implies

$$
\gamma_{p}^{p+1} \leq(2 M)^{2 p} / 2 \pi
$$

We finally conclude $\gamma_{p} \leq C_{2}$ for some $C_{2}$ independent of large $p$.

We include a consequence of Theorem 1.1 here which will be used later.

Corollary 3.1. There exist positive $C_{1}$ and $C_{2}$ such that

$$
\frac{C_{1}}{p} \leq \int_{\Omega} u_{p}^{p} \leq \frac{C_{2}}{p}
$$

Proof. The first inequality follows from Theorem 1.1 and the first limit of Corollary 2.3 and the second inequality follows from the first limit of Corollary 2.3 through an interpolation.

\section{Proof of TheOrem 1.2}

We first state a boundary estimate lemma. The proof of the lemma is standard. One combines the moving plane method in [5] with a Kelvin transform. We refer to [3] and [5] for details. 
Lemma 4.1. Let $u$ be a solution of

$$
\begin{aligned}
& \Delta u+f(u)=0 \quad \text { in } \Omega \subset R^{2}, \\
& \left.u\right|_{\partial \Omega}=0, \quad u>0 \text { in } \Omega
\end{aligned}
$$

where $\Omega$ is bounded, smooth and $f$ is a smooth function. Then there exist a neighborhood $\omega$ of $\partial \Omega$ and a constant $C$ both depending on the geometry of $\Omega$ only such that $\|u\|_{L^{\infty}(\omega)} \leq C\|u\|_{L^{1}(\Omega)}$.

Applying this lemma to $v_{p}=u_{p} / \int_{\Omega} u_{p}^{p}$ we have the following uniform boundary estimate. In particular, it implies that $\left\{v_{p}\right\}$ does not blow up on the boundary of $\Omega$; hence $\left\{u_{p}\right\}$ has no peak on the boundary for any peak point of $\left\{u_{p}\right\}$ must be a blow-up point of $\left\{v_{p}\right\}$; see Remark 4.7.

Lemma 4.2. There exist a constant $C$ and a neighborhood $\omega$ of $\partial \Omega$ both depending on the geometry of $\Omega$ only such that $v_{p} \leq C$ in $\omega$.

Proof. Because $\left\|\Delta v_{p}\right\|_{L^{1}}=1$, combining the elliptic $L^{p}$ estimate with the duality argument (see [2] for details), we have that $v_{p}$ is bounded uniformly in $W^{1, q}(\Omega)$ for $1 \leq q<2$; hence $v_{p}$ is uniformly bounded in $L^{1}$. Then using Lemma 4.1, we obtain the desired result.

We quote some interesting results from [1]. The first one is their Theorem 1, while the second one is a combination of their Corollary 3 and Corollary 4 .

Lemma 4.3. Let $u$ be a solution of

$$
-\Delta u=f \quad \text { in } \Omega,\left.\quad u\right|_{\partial \Omega}=0
$$

where $\Omega$ is a smooth bounded domain in $R^{2}$. We have for $0<\epsilon<4 \pi$

$$
\int_{\Omega} \exp \left[\frac{(4 \pi-\epsilon)|u(x)|}{\|f\|_{L^{1}}}\right] d x \leq \frac{4 \pi^{2}}{\epsilon}(\operatorname{diam} \Omega)^{2} .
$$

Lemma 4.4. Let $u_{n}$ be a sequence of solutions of

$$
\Delta u_{n}+V_{n} e^{u_{n}}=0 \quad \text { in } \Omega,\left.\quad u_{n}\right|_{\partial \Omega}=0
$$

such that $\left\|V_{n}\right\|_{L^{q}} \leq C$ for some $1<q<\infty$ and

$$
\int_{\Omega}\left|V_{n}\right| e^{u_{n}} \leq \epsilon_{0}<\frac{4 \pi}{q^{\prime}}
$$

where $q^{\prime}$ is the Hölder conjugate of $q$ for all $n$. Then $\left\{u_{n}\right\}$ is uniformly bounded in $L^{\infty}(\Omega)$.

Let $u_{n}$ be a sequence of solutions to equations $\Delta u+V_{n} e^{u}=0$ in $\Omega \subset R^{2}$ with no boundary condition. Assume $\left\|u_{n}^{+}\right\|_{L^{1}} \leq C,\left\|V_{n}\right\|_{L^{q}} \leq C$ for some $1<q<\infty$ and $\int_{\Omega}\left|V_{n}\right| e^{u_{n}} \leq \epsilon_{0} 4 \pi / q^{\prime}$ where $q^{\prime}$ is the Hölder conjugate of $q$ for all $n$. Then $\left\{u_{n}^{+}\right\}$is uniformly bounded in $L_{l o c}^{\infty}(\Omega)$.

Now recall

$$
L_{0}=\varlimsup_{p \rightarrow \infty} \frac{p \nu_{p}}{e}
$$

where $\nu_{p}=\int_{\Omega} u_{p}^{p}$. 
We denote any sequence $u_{p_{n}}$ of $u_{p}$ by $u_{n}$ and $\nu_{p_{n}}$ of $\nu_{p}$ by $\nu_{n}$. Because $u_{n}$ has the property

$$
\int_{\Omega} \frac{u_{n}^{p_{n}}}{\int_{\Omega} u_{n}^{p_{n}}}=1,
$$

we can subtract a subsequence of $u_{n}$, still denoted by $u_{n}$, such that there is a positive bounded measure $\mu$ in $M(\Omega)$, the set of all real bounded Borel measures on $\Omega$, such that $\mu(\Omega) \leq 1$ and

$$
\int_{\Omega} f_{n} \varphi \rightarrow \int_{\Omega} \varphi d \mu
$$

for all $\varphi \in C_{0}(\Omega)$ where

$$
v_{n}=u_{n} / \nu_{n} \quad \text { and } \quad f_{n}=\nu_{n}^{p_{n}-1} v_{n}^{p_{n}} .
$$

For any $\delta>0$, we call $x_{0}$ is a $\delta$-regular point if there is a function $\varphi$ in $C_{0}(\Omega), 0 \leq \varphi \leq 1$, with $\varphi=1$ in a neighborhood of $x_{0}$ such that

$$
\int_{\Omega} \varphi d \mu<\frac{4 \pi}{L_{0}+2 \delta} \text {. }
$$

We define

$$
\Sigma(\delta)=\left\{x_{0} \in \Omega: x_{0} \text { is not a } \delta \text {-regular point }\right\} .
$$

We shall frequently say 'regular', 'irregular', and ' $\Sigma$ ' not mentioning $\delta$ if no confusion exists.

Lemma 4.5. Let $S$ be the blow-up set defined in (1.5) of the subsequence $v_{n}$. Then $S$ is nonempty and there is a small neighborhood $\omega$ of $\partial \Omega$ which depends on the geometry of $\Omega$ only such that $S$ does not contain any point in $\omega$.

Proof. The second assertion follows immediately from Lemma 4.2. For the first one, observe

$$
\max v_{n}(x) \geq \frac{C}{\nu_{n}} \rightarrow \infty
$$

from Theorem 1.1, Corollary 3.1, and the definition of $v_{n}$.

Our next lemma plays a central role in the proof of Theorem 1.2. It says that smallness of $\mu$ at a point $x_{0}$ implies boundedness of $v_{n}$ near $x_{0}$.

Lemma 4.6. If $x_{0}$ is a $\delta$-regular point, then $\left\{v_{n}\right\}$ is bounded in $L^{\infty}\left(B_{R_{0}}\left(x_{0}\right)\right)$ for some $R_{0}>0$.

Proof. Let $x_{0}$ be a regular point. From the definition of regular points, there exists $R_{1}>0$ such that

$$
\int_{B_{R_{1}}\left(x_{0}\right)} f_{n}<\frac{4 \pi}{L_{0}+\delta} .
$$

Split $v_{n}$ into two parts, $v_{n}=v_{1 n}+v_{2 n}$, where $v_{1 n}$ is the solution of

$$
\Delta v_{1 n}+f_{n}=0 \quad \text { in } B_{R_{1}}\left(x_{0}\right),\left.\quad v_{1 n}\right|_{\partial B_{R_{1}}\left(x_{0}\right)}=0
$$

and $v_{2 n}$ solves

$$
\Delta v_{2 n}=0 \quad \text { in } B_{R_{1}}\left(x_{0}\right),\left.\quad v_{2 n}\right|_{\partial B_{R_{1}}\left(x_{0}\right)}=\left.v_{n}\right|_{\partial B_{R_{1}}\left(x_{0}\right)} .
$$


From the maximum principle, $v_{1 n} v_{2 n}>0$. By the mean value theorem for harmonic functions, we have

$$
\left\|v_{2 n}\right\|_{L^{\infty}\left(B_{R_{1} / 2}\right)} \leq C\left\|v_{2 n}\right\|_{L^{1}\left(B_{R_{1}}\right)} \leq C\left\|v_{n}\right\|_{L^{1}(\Omega)} \leq C
$$

where the last inequality follows as in the proof of Lemma 4.2. So we need only to consider $v_{1 n}$. To estimate $v_{1 n}$ we would like to apply Lemma 4.4. From

Let $t$ be such that $t^{\prime}=L_{0}+\delta / 2$ where $t^{\prime}$ is the Hölder conjugate of $t$.

$$
\int_{B_{R_{1}}} f_{n}<\frac{4 \pi}{L_{0}+\delta}
$$

using Lemma 4.3, we have

$$
\int_{B_{R_{1}}\left(x_{0}\right)} \exp \left[\left(L_{0}+\delta / 2\right)\left|v_{1 n}(x)\right|\right] \leq C
$$

if we choose $\epsilon$ in Lemma 4.3 small enough.

Now observe $\log x \leq x / e$ for $x>0$. We have

$$
p_{n} \log \frac{u_{n}}{\nu_{n}^{1 / p_{n}}} \leq \frac{p_{n}}{e} \frac{u_{n}}{\nu_{n}^{1 / p_{n}}} \leq \frac{L_{0}+\delta / 3}{\nu_{n}} \frac{u_{n}}{\nu_{n}^{1 / p_{n}}} \leq \frac{t^{\prime}-\delta / 6}{\nu_{n}^{1 / p_{n}}} \frac{u_{n}}{\nu_{n}} \leq t^{\prime} \frac{u_{n}}{\nu_{n}}
$$

for $n$ large enough because

$$
\lim _{n \rightarrow \infty} \nu_{n}^{1 / p_{n}}=1
$$

which follows from Corollary 3.1. Hence

$$
f_{n} \leq e^{t^{\prime} v_{n}}, \quad\left(f_{n}\right)^{t} e^{-t v_{1 n}} \leq e^{\left(t^{\prime}+t\right) v_{2 n}+t^{\prime} v_{1 n}} .
$$

Therefore on $B_{R_{1} / 2}\left(x_{0}\right)$ since $v_{2 n}$ is uniformly bounded, we have

$$
\left(f_{n}\right)^{t} e^{-t v_{1 n}} \leq C e^{t^{\prime} v_{1 n}}
$$

on $B_{R_{1} / 2}\left(x_{0}\right)$. Combining (4.6) and (4.7), we get that $f_{n} e^{-v_{1 n}}$ is bounded in $L^{t}\left(B_{R_{1 / 2}\left(x_{0}\right)}\right)$. Therefore applying Lemma 4.4 part 2 to $v_{1 n}$ on $B_{R_{1} / 2}\left(x_{0}\right)$, we get uniform $L^{\infty}$ bound for $\left\{v_{1 n}\right\}$; hence uniform $L^{\infty}$ bound for $\left\{v_{n}\right\}$ on $B_{R_{1} / 4}\left(x_{0}\right)$.

We finally get down to the goal of this section.

Proof of Theorem 1.2. We first claim $S=\Sigma(\delta)$ for any $\delta>0$.

Clearly $S \subset \Sigma$. In fact, let $x_{0} \notin \Sigma$; then $x_{0}$ is a regular point. Hence by Lemma $4.6\left\{v_{n}\right\}$ is bounded in $L^{\infty}\left(B_{R}\left(x_{0}\right)\right)$ for some $R$, i.e., $x_{0} \notin S$.

Conversely, suppose $x_{0} \in \Sigma$. Then we have for every $R>0$, passing to a subsequence of $\left\{v_{n}\right\}$ if necessary,

$$
\lim _{n \rightarrow \infty}\left\|v_{n}\right\|_{L^{\infty}\left(B_{R}\left(x_{0}\right)\right)}=\infty
$$

Otherwise there would be some $R_{0}>0$ such that $\left\|v_{n}\right\|_{L^{\infty}\left(B_{R_{0}}\left(x_{0}\right)\right)}<C$ for some $C$ independent of $n$. Then

$$
f_{n}=\nu_{n}^{p_{n}-1} v_{n}^{p_{n}} \leq\left(M / p_{n}\right)^{p_{n}-1} C^{p_{n}} \rightarrow 0
$$


uniformly as $n \rightarrow \infty$ on $B_{R_{0}\left(x_{0}\right)}$. Then

$$
\int_{B_{R_{0}}\left(x_{0}\right)} f_{n}=\int_{B_{R_{0}}\left(x_{0}\right)} \nu_{n}^{p_{n}-1} v_{n}^{p_{n}} \leq \epsilon_{0}<\frac{4 \pi}{L_{0}+2 \delta}
$$

which implies that $x_{0}$ is a regular point, i.e., $x_{0} \notin \Sigma$. Contradiction. Equation (4.8) then implies by the definition (1.5) of $S$ that $x_{0} \in S$. This completes the proof of our claim.

Next we go back to measure $\mu$ defined in (4.1). Clearly by (4.2)

$$
1 \geq \mu(\Omega) \geq \frac{4 \pi}{L_{0}+2 \delta} \# \Sigma(\delta)=\frac{4 \pi}{L_{0}+2 \delta} \# S .
$$

Hence combining this with Lemma 4.5

$$
1 \leq \# S \leq\left(L_{0}+2 \delta\right) / 4 \pi \text {. }
$$

Applying Lemma 2.4, we finally conclude that by choosing $\delta$ small $1 \leq \# S \leq 2$ for arbitrary bounded smooth domains and $\# S=1$ for domains with condition (T).

Remark 4.7. The fact that the peak set of $\left\{u_{n}\right\}$ is included in the blow-up set $S$ of $\left\{v_{n}\right\}$ follows easily from the fact that $\nu_{n} \rightarrow 0$ as $n \rightarrow \infty$ by Corollary 3.1 .

\section{Proof of Theorem 1.3}

From Lemma 4.2 and Lemma 4.5, we have that

$$
v_{n} \leq C
$$

on any compact $K \subset \bar{\Omega} \backslash\left\{x_{0}\right\}$. Lemma 2.4 then implies $f_{n} \rightarrow 0$ uniformly on any compact $K \subset \bar{\Omega} \backslash\left\{x_{0}\right\}$.

Take $\varphi \in C_{0}(\Omega)$. We have for any $\epsilon$

$$
\begin{aligned}
& \left|\int_{\Omega}\left[f_{n} \varphi d x-\varphi\left(x_{0}\right)\right] d x\right| \leq \int_{\Omega} f_{n}\left|\varphi(x)-\varphi\left(x_{0}\right)\right| d x \\
& \quad \leq \int_{B_{\delta}\left(x_{0}\right)} f_{n}\left|\varphi(x)-\varphi\left(x_{0}\right)\right| d x+\int_{\Omega \backslash B_{\delta}\left(x_{0}\right)} f_{n}\left|\varphi(x)-\varphi\left(x_{0}\right)\right| d x \\
& \quad \leq \epsilon / 2+\epsilon / 2=\epsilon
\end{aligned}
$$

if we first choose $\delta$ small enough and then choose $n$ large enough. This proves part (1).

On any compact $K \subset \bar{\Omega} \backslash\left\{x_{0}\right\}$, because $v_{n}$ are bounded and $f_{n} \rightarrow 0$ uniformly, we have by the elliptic regularity theory a subsequence of $v_{n}$, still denoted by $v_{n}$ that approaches a function, say $G^{\prime}$ in $C^{2, \alpha}(K)$, weakly in $W^{1, q}(\Omega)$ and strongly in $L^{1}(\Omega)$ while the second and the third convergence comes from the $W^{1, q}(\Omega)$ boundedness of $v_{n}$ for $1<q<2$ [2] and the compactness of the embedding $W^{1, q}(\Omega) \hookrightarrow L^{1}(\Omega)$.

Then for any $\varphi \in C_{0}^{\infty}(\Omega)$

$$
-\int_{\Omega} G^{\prime} \Delta \varphi d x=-\lim _{n \rightarrow \infty} \int_{\Omega} v_{n} \Delta \varphi=-\lim _{n \rightarrow \infty} \int_{\Omega} \Delta v_{n} \varphi=\lim _{n \rightarrow \infty} \int_{\Omega} f_{n} \varphi=\varphi\left(x_{0}\right)
$$

from part (1). Therefore $G^{\prime}=G\left(, x_{0}\right)$. We proved part (2). 
To prove part (3) we assume, without loss of generality, $x_{0}=0$. We need only to prove that 0 is a critical point of function $R$ defined in Theorem 1.3.

By Pohozaev's Identity (2.3), we have for any $u$ that solves (1.2)

$$
\frac{2}{p+1} \int_{\Omega} u^{p+1}=\frac{1}{2} \int_{\partial \Omega}(x-y, n)\left(\frac{\partial u(x)}{\partial n(x)}\right)^{2} d s
$$

for all $y \in R^{2}$. Therefore

$$
\frac{1}{2} \int_{\partial \Omega} n(x)\left(\frac{\partial u(x)}{\partial n(x)}\right)^{2} d s=0
$$

where 0 in the equation is the zero vector in $R^{2}$.

Now let $u=v_{n}$ in (5.2) and pass the limit. By the boundary regularity proved in $(5.1)$, we have

$$
\int_{\partial \Omega} n(x)\left(\frac{\partial G(x, 0)}{\partial n(x)}\right)^{2} d s=0 .
$$

Let us state a lemma. Part (3) of the theorem will follow immediately.

\section{Lemma 5.1.}

$$
\int_{\partial \Omega} n(x)\left(\frac{\partial G(x, 0)}{\partial n(x)}\right)^{2} d s=-\nabla R(0)
$$

where $R(x)=g(x, x)$ and

$$
G(x, y)=g(x, y)-\frac{1}{2 \pi} \log |x-y| .
$$

Proof. Applying Pohozaev's Identity (2.3) to

$$
G(x, 0)=g(x, 0)-\frac{1}{2 \pi} \log |x|
$$

on $\Omega \backslash B_{r}(0)$, we get

$$
\begin{aligned}
\int_{\partial \Omega} & {\left[(x-y, \nabla G(x, 0)) \frac{\partial G(x, 0)}{\partial n}-(x-y, n) \frac{|\nabla G(x, 0)|^{2}}{2}\right] d s } \\
& =\int_{\partial B_{r}}\left[(x-y, \nabla G(x, 0)) \frac{\partial G(x, 0)}{\partial n}-(x-y, n) \frac{|\nabla G(x, 0)|^{2}}{2}\right] d s
\end{aligned}
$$

for all $y \in R^{2}$. Therefore

$$
\begin{aligned}
\int_{\partial \Omega} & {\left[\nabla G(x, 0) \frac{\partial G(x, 0)}{\partial n}-\frac{|\nabla G(x, 0)|^{2}}{2} n(x)\right] d s } \\
= & \int_{\partial B_{r}}\left[\nabla G(x, 0) \frac{\partial G(x, 0)}{\partial n}-\frac{|\nabla G(x, 0)|^{2}}{2} n(x)\right] d s .
\end{aligned}
$$

Notice on $\partial \Omega$

$$
\nabla G(x, 0)=\frac{\partial G(x, 0)}{\partial n} n(x)
$$


We have

$\frac{1}{2} \int_{\partial \Omega} n(x)\left(\frac{\partial G(x, 0)}{\partial n}\right)^{2} d s=\int_{\partial B_{r}}\left[\nabla G(x, 0) \frac{\partial G(x, 0)}{\partial n}-\frac{|\nabla G(x, 0)|^{2}}{2} n(x)\right] d s$

where the right-hand side equals

$$
\begin{aligned}
\mathrm{RHS}= & \int_{\partial B_{r}}\left[\nabla\left(g(x, 0)-\frac{1}{2 \pi} \log |x|\right) \frac{\partial\left(g(x, 0)-\frac{1}{2 \pi} \log |x|\right)}{\partial n}\right. \\
& \left.\quad-\frac{n(x)}{2}\left|\nabla\left(g(x, 0)-\frac{1}{2 \pi} \log |x|\right)\right|^{2}\right] d s \\
= & \int_{\partial B_{r}}\left[\nabla g \frac{\partial g}{\partial n}-\nabla g \frac{1}{2 \pi} \frac{\partial \log |x|}{\partial n}\right. \\
& \quad-\frac{1}{2 \pi} \nabla \log |x| \frac{\partial g}{\partial n}+\frac{1}{4 \pi^{2}} \nabla \log |x| \frac{\partial \log |x|}{\partial n} \\
& \left.\quad-\frac{n(x)}{2}|\nabla g|^{2}+n(x)\left(\nabla g, \frac{1}{2 \pi} \nabla \log |x|\right)-\left.\frac{n(x)}{2} \frac{1}{4 \pi^{2}}|\nabla \log | x\right|^{2}\right] d s \\
= & \int_{B_{r}}\left[\nabla g \frac{\partial g}{\partial n}-\frac{n(x)}{2}|\nabla g|^{2}-\frac{1}{2 \pi} \nabla \log |x| \frac{\partial g}{\partial n} n(x)\right] d s \\
= & I_{1}-I_{2}-I_{3} .
\end{aligned}
$$

As $r \rightarrow 0, I_{1}$ and $I_{2}$ both approach 0 while

$$
\left.I_{3} \rightarrow \nabla g(x, 0)\right|_{x=0}=\frac{1}{2} \nabla R(0) \text {. }
$$

This proves the lemma, hence in turn, Theorem 1.3.

Theorem 1.3 has an extension to the case where $S$ contains two points. But we suspect that the double-peak asymptotic behavior would not happen for arbitrary smooth bounded domain. Examining the proof of Theorem 1.2, we see that the only place where the estimate is rough is the place where we estimate $L_{0}$. Actually it is the Lemma 2.4 that might need to be improved. So we would like to propose the following.

Conjecture 5.2. For any smooth bounded domain in $R^{2}$ any sequence $u_{p_{n}}$ of the solutions to (1.2) obtained by the variational method with nonlinear exponent equal to $p_{n}$, after passing to a subsequence, must develop a single peak when $p_{n}$ approaches infinity.

One way to prove this conjecture is to prove

Conjecture 5.3. For any smooth bounded domain in $R^{2}$ let $L_{0}=\varlimsup_{\lim _{p \rightarrow \infty}}\left(p \nu_{p} / e\right)$ where $\nu_{p}=\int_{\Omega} u_{p}^{p}$ and $u_{p}$ are solutions of (1.2) obtained by the variational method. Then we have $L_{0}<8 \pi$.

\section{ACKNOWLEDGMENT}

The authors would like to thank their adviser, Professor Wei-Ming Ni, for bringing solutions with spiky patterns to their attention. 
Note added in proof. The authors have recently confirmed Conjecture 5.2 . This result will appear in a forthcoming paper.

\section{REFERENCES}

1. H. Brezis and F. Merle, Uniform estimate and blow-up behavior for solutions of $-\Delta u=$ $V(x) e^{u}$ in two dimensions, Comm. Partial Differential Equations 16 (1991), 1223-1253.

2. H. Brezis and W. Strauss, Semilinear second-order elliptic equations in $L^{1}$, J. Math. Soc. Japan 25 (1973), 565-590.

3. D. G. DeFigueiredo, P. L. Lions, and R. D. Nussbaum, A priori estimates and existence of positive solutions of semilinear elliptic equations, J. Math. Pures. Appl. 61 (1982), 41-63.

4. H. Federer, Geometric measure theory, Springer-Verlag, Berlin-Heidelberg-New York, 1969.

5. B. Gidas, W.-M. Ni, and L. Nirenberg, Symmetry and related properties via the maximum principle, Comm. Math. Phys. 68 (1979) 209-243.

6. D. Gilbarg and S. N. Trudinger, Elliptic partial differential equations of second order, 2nd ed., Springer-Verlag, Berlin-Heidelberg-New York, 1983.

7. Z. Han, Asymptotic approach to singular solutions for nonlinear elliptic equations involving critical Sobolev exponent, Analyse Nonlinéaire 8-2 (1991).

8. J. Moser, A sharp form of an inequality by Trudinger, Indiana Univ. Math. J. 20 (1971), 1077-1092.

9. W.-M. Ni and I. Takagi, On the shape of least-energy solutions to a semilinear Neumann problem, Comm. Pure. Appl. Math. 44 (1991).

10. S. Pohozaev, Eigenfunctions of the equation $\Delta u+\lambda f(u)=0$, Soviet Math. Dokl. 6 (1965), 1408-1411.

11. X. Ren and J. Wei, On a semilinear elliptic problem in $R^{2}$ when the exponent approaches infinity, preprint.

12. O. Rey, The role of the Green's function in a nonlinear elliptic equation involving the critical Sobolev exponent, J. Funct. Anal. 89 (1990), 1-52.

School of Mathematics, University of Minnesota, Minneapolis Minnesota 55455

E-mail address: ren@math.umn.edu

E-mail address: wei@math.umn.edu 